\title{
Effect of Astaxanthin Supplementation on Antioxidant Capacity, Blood Lactate and Blood Uric Acid Metabolism in Human Recovery Stage After Exercise
}

\author{
Lijun Wu ${ }^{1}$, Zhuo Sun ${ }^{1,}$, , Jing Zhao ${ }^{1}$, Xinming Guo ${ }^{2}$, Jie Wang ${ }^{1}$ \\ ${ }^{1}$ Department of Human Kinesiology, Physical Education of Shanxi University, Taiyuan, China \\ ${ }^{2}$ Department of Physical Education, Taiyuan Normal College, Taiyuan, China
}

Email address:

980149186@qq.com (Zhuo Sun)

${ }^{*}$ Corresponding author

\section{To cite this article:}

Lijun Wu, Zhuo Sun, Jing Zhao, Xinming Guo, Jie Wang. Effect of Astaxanthin Supplementation on Antioxidant Capacity, Blood Lactate and Blood Uric Acid Metabolism in Human Recovery Stage After Exercise. Advances in Bioscience and Bioengineering.

Vol. 7, No. 4, 2019, pp. 60-63. doi: 10.11648/j.abb.20190704.11

Received: September 2, 2019; Accepted: September 23, 2019; Published: October 9, 2019

\begin{abstract}
To study the effects of astaxanthin supplementation on serum antioxidant capacity, blood lactic acid and blood uric acid metabolism of human body in the $1 \mathrm{~h}$ recovery period after acute high-intensity exercise. Sixteen male students grade 2016 from Physical Education Institution (age: $19.13 \pm 2.52$, height: $179 \pm 4.21 \mathrm{~cm}$ and weight $68.23 \pm 7.81 \mathrm{~kg}$ ) were randomly divided into two groups: group A (experimental) and B (control). Group A was given astaxanthin for 28 days at a moderate dose $(12 \mathrm{mg} / \mathrm{d})$ and placebo for 28 days at group B. 4 weeks later, all subjects underwent exercise on power bike. The antioxidant capacity and blood uric acid and the content of blood lactic acid was assayed using anti-oxidation Kit (FORD), blood lactic acid Kit (LACTIC ACID), blood uric acid Kit (URIC ACID). Results: 1) One hour after exercise, the antioxidant capacity in both A and B obviously increased without exception and the value of group A was obviously higher than B. The blood lactic acid in both groups dramatically declined and the value of group A was obviously lower than B. The blood uric acid rised in both groups and the value of A was obviously lower than B. Conclusions: Pre-supplement of astaxanthin can accelerate the recovery of antioxidant capacity, accelerate the clearance of blood lactate and delay the increase of blood uric acid in the body within 1 hour after exercise.
\end{abstract}

Keywords: Astaxanthin, Exercise Recovery, Antioxidant Capacity, Blood Lactic Acid, Blood Uric Acid

\section{Introduction}

The metabolism of cells in living organisms is accompanied by the generation of free radicals such as reactive oxygen species [1-2]. Free radicals are extremely unstable and can react with lipids, proteins and DNA in the body [3]. Excessive free radicals can cause imbalance between oxidation and antioxidants in the body, which can lead to disease.

Astaxanthin (ASTA) is a kind of lutein carotenoid found in marine organisms (fish, shrimp, algae) [4], which can scavenge free radicals by attracting free radicals or supplying electrons to free radicals. Therefore, it can inhibit damage caused by cellular lipids and DNA peroxidation, and astaxanthin has a positive effect on relieving eye fatigue, anti-inflammatory, and protecting the central nervous system. In the field of sports science, astaxanthin has the effect of relieving skeletal muscle and myocardial damage, maintaining skeletal muscle tone and endurance, and delaying exercise fatigue [5-7]. In recent years, there has been some new research on astaxanthin in the field of sports. Ayano's research shows that astaxanthin supplement has significant improvement in subjective feelings, work efficiency and autonomic activity in patients with physical and mental fatigue [8]. Maki Takami's study showed that astaxanthin supplementation decreased levels of serum insulin after $30 \mathrm{~min}$ moderate-intensity power cycle training and indicate that astaxanthin could modulate training-induced aerobic metabolism of carbohydrate and fat during rest and exercise [9]. Pre-supplement of astaxanthin for 4 weeks has 
not been reported on the body's antioxidant capacity, blood lactic acid and blood uric acid metabolism in the recovery period after acute high-intensity exercise. Our research will focus on this issue.

\section{Materials and Methods}

\subsection{Grouping of Experimental Objects}

Sixteen male students grade 2016 from Physical Education Institution (age: $19.13 \pm 2.52$, height: $179 \pm 4.21 \mathrm{~cm}$ and weight $68.23 \pm 7.81 \mathrm{~kg})$. They were randomly divided into two groups ( $\mathrm{n}=8$ per group): experimental group A (Supplementary medium dose astaxanthin: $12 \mathrm{mg} / \mathrm{d}$ ) and control group $\mathrm{B}$ (Placebo). Sixteen subjects were asked the medical history and undergo a routine physical examination to confirm their health and no major disease or suffer upper respiratory tract infections, cardiovascular, Otherwise they were not intake supplements as free radical scavenger (VE, VC, lycopene) and traditional Chinese medicine recently. This clinical trial followed the Declaration of Helsinki, and informed consent was obtained from the subjects before the experiment.

\subsection{Laboratory Instruments and Reagents}

Sweden made Monark874E quantitative load power cycle was using to complete acute exercise. The antioxidant capacity and blood uric acid and the content of blood lactic acid was measured by the Italy CR3000RC type oxygen free radical biochemistry analyzer, centrifuge, adjustable pipette, anti-oxidation Kit (FORD), blood lactic acid Kit (LACTIC ACID), blood uric acid Kit (URIC ACID) (kits were produced from Italy Callegari company). The astaxanthin in this study supplied by Hubei astaxanthin Biotech Inc ASTA astaxanthin soft capsule $500 \mathrm{mg} /$ tablets (each containing astaxanthin is larger than $4 \mathrm{mg}$, Hubei food key word: 2012 Article No. 0014).

\subsection{Experimental Design and Motion Scheme}

The experiment was set 29 days. The experimental group supplement astaxanthin in doses of $12 \mathrm{mg}$ at 21:00 $7 \mathrm{~d}$ a week for 4 weeks. The control group taking the Placebo as similar appearance as astaxanthin for 4 weeks too. 28 days after the drug supplement, in the next morning at $8 \mathrm{AM}$, all the participants taking acute exercise immediately on pedal power bicycle with all strength 30 seconds $\times 3 / 3 \mathrm{~min}$ interval (loading a weight of $0.075 \mathrm{~kg} / \mathrm{kg}$ ) [10]. At the end of the exercise, all subjects performed first blood taking, and second blood samples was taking one hour after exercise. Each blood collection was using capillary microtubules on the fingertip, and the antioxidant capacity, blood lactate and serum uric acid blood samples were tested instantly [11].

\subsection{Test Indexes and Methods}

\subsubsection{Test of Antioxidant Capacity (FORD)}

Testing fundamentals: The FORD test is the chromogenic reaction (4-diethyl-aminoaniline sulfate included) that an oxidizing agent (Ferric chloride) in acid environment $(\mathrm{pH}=5.2)$ forms the corresponding cation radical (Chromogenic substrate + ). The stability and color of this type of amino acid can be measured at $505 \mathrm{~nm}$. Adding antioxidant compounds $(\mathrm{AOH})$ to the sample reduces the amount of cationic free radical amino acids and also make the color loss proportional. The absorbance value of concentration was measured through the law of LambertBeer.

(1) Chromogenic substrate $+($ colorless $)+\mathrm{Fe}^{3+}+\mathrm{H}^{+}$ $\rightarrow$ Chromogenic substrate + (red)

(2) Chromogenic substrate + (red) $+\mathrm{AOH} \rightarrow$ Chromogenic substrate + (colorless $)+\mathrm{AO}$

\subsubsection{Blood Lactic Acid Test (LACTIC ACID)}

Testing fundamentals: The lactic acid is oxidized to pyruvate and hydrogen peroxide under the action of lactic acid oxidase (LOD) (action 1). A red compound was composed in the presence of peroxidase and catalyzed by hydrogen peroxide, 4- aminoatipyrine (4-AP) and phenolic derivatives (action 2). Reading the compound intensity of such color at $505 \mathrm{~nm}$ and directly proportional to the concentration of lactic acid in the sample.

(1) Lactic acid Lacticacidoxidase $\longrightarrow$ Pyruvate $+\mathrm{H}_{2} \mathrm{O}_{2}$

(2) $\mathrm{H}_{2} \mathrm{O}_{2}+4$-aminoatipyrine (4-AP) + Phenolic derivative Peroxidase $\longrightarrow$ Compound (red) $+\mathrm{H}_{2} \mathrm{O}_{2}$

\subsubsection{Uric Acid Test (URIC ACID)}

Testing fundamentals: Uric acid can produce uric acid allantoin and hydrogen peroxide in the role of uric acid oxidase (action 1). Hydrogen peroxide, 4-amino antipyrine and phenol derivatives form a red compound and water under the action of peroxidase (action 2). Reading at $505 \mathrm{~nm}$ of the compound intensity of such color is directly proportional to the concentration of uric acid in the sample.

(1) Uric acid Acid oxidase $\longrightarrow$ Uric acid allantoin $+\mathrm{H}_{2} \mathrm{O}_{2}$

(2) $\mathrm{H}_{2} \mathrm{O}_{2}+4$-aminoatipyrine (4-AP) + Phenolic derivative Peroxidase $\longrightarrow$ Compound (red) $+\mathrm{H}_{2} \mathrm{O}_{2}$ [11]

\subsection{Experimental Results Processing}

SPSS for Windows v22.0 was used for statistical analyses. Comparative the two groups using independent sample Ttest, and per-post each group were compared with paired samples T-test, $\mathrm{P}<0.05$ for the difference was significant, and $\mathrm{P}<0.01$ was considered significant. A value of $\mathrm{p}<0.05$ was accepted as statistically significant, and $p<0.01$ was striking significant.

\section{Results}

Effects of $1 \mathrm{~h}$ Restore After Acute High-intensity Exercise on Body Anti-oxidation Ability Value, Blood Lactate, Uric Acid

One hour after exercise, all subjects showed a significant increase in anti-oxidative capacity values $(\mathrm{P}<0.05)$ and the experimental group was significantly higher than control 
group $(\mathrm{P}<0.01)$. Blood lactate values were significantly decreased $(\mathrm{P}<0.01)$, but the experimental group was significantly lower than the control group $(\mathrm{P}<0.05)$.
However, in experimental group a significant increase in uric acid $(\mathrm{P}<0.01)$ and evident lower than the control group $(\mathrm{P}<0.05)$ (Table 1).

Table 1. Change of antioxidant capacity of human body, blood lactic acid and blood uric acid one hour after exercise.

\begin{tabular}{llll}
\hline & & Antioxidant capacity (mM) & blood lactic acid (mM) \\
\hline \multirow{2}{*}{ Control group $(\mathrm{n}=8)$} & Previous & $0.60 \pm 0.19$ & $5.71 \pm 0.72$ \\
& After & $0.67 \pm 0.18^{\#}$ & $3.92 \pm 1.18^{\# \#}$ \\
& Previous & $0.83 \pm 0.22$ & $4.97 \pm 0.60$ \\
Experimental group $(\mathrm{n}=8)$ & After & $0.95 \pm 0.18^{* * \#}$ & $2.72 \pm 0.56^{* \# \#}$ \\
\hline
\end{tabular}

Note: Each value represents mean $\pm \mathrm{SD} * \mathrm{P}<0.05$ vs control group, $* * \mathrm{P}<0.01$ vs control group; \# $\mathrm{P}<0.05 \mathrm{vs}$ before restore, \#\# $<<0.01 \mathrm{vs}$ before restore.

\section{Discussion}

Effect on Body Anti-oxidation Ability Value, Blood Lactate, Uric Acid of 1 h Restore After Acute High-intensity Exercise

Seen from table 1 , the recovery period after exercise, antioxidation ability value of subjects were increased in two groups, and the experimental group recover faster than the control group. In this case the free radical content is reducing, which descript astaxanthin can promote the body's anti-oxidant recovery. From the general trend, experiment group has a significant advantage than control group in antioxidation ability at before and after exercise and the recovery period after exercise. It suggests that taking moderate doses of astaxanthin has a positive improvement on the body at rest, the acute anti-high-intensity exercise before and after oxidation. Body antioxidant capacity and the health level is positively related, indicating that astaxanthin in maintaining body's health, improving the body's antioxidant defense system capacity and thus has a good effect on prevention of damage to the body.

1 hour after exercise, blood lactate of two groups were significantly decreased $(\mathrm{P}<0.01)$, with the experimental group significantly lower than the control group $(\mathrm{P}<0.05)$, but the value did not return to the quiet state, which may be related to the insufficient recovery time. Table 1 shows that blood lactate in experimental group recovery faster than the control group and closer to resting blood lactate. Suggesting that astaxanthin can promote blood lactate clearance after exercise. Katrin observed that lactic acid accumulates under the inflammatory conditions and affects the function of local immune cells. He comprehensively analyzed the effect of lactic acid on gene expression during monocyte activation, and concluded that lactate has a profound effect on gene expression and function of human monocytes, which can inhibit the monocyte's role in the tumor environment [12]. Visible, lactic acid will have an extremely adverse effect on the body. In this study, astaxanthin on the role of lactic acid in the body: lactic acid content of the body can be reduced in advance in a quiet state; inhibit the production of lactic acid caused by the acute high-intensity exercise; it can accelerated the removal of the lactic acid in the body after exercise.

Yuichiro and other studies found that moderate exercise and obesity are related to lower levels of uric acid, and no evidence shows the correlation between high intensity and low uric acid concentration [13]. Lamina studies have shown that moderate-intensity exercise can reduce hypertensive patients' blood pressure, serum uric acid level and psychosocial stress after 8 weeks training [14]. Huang and other studies found that exercise in a high-temperature which caused a lot of sweat will reduce the uric acid excretion and increase blood uric acid after exercise at least 1 hour. That's mainly due to the reduction in effective renal blood flow. We recommended sufficiently fluid supplementation to prevent dehydration and maintain adequate urine amount [15]. This study arranged by acute high intensity exercise, and 1 hour later, the uric acid has a significantly increased in both groups $(\mathrm{P}<0.01)$. These results are similar to Huang's findings. The possible reasons may as follows, (1) exercise can induced renal blood flow and filtration rate, thus causing the decrease in glomerular filtration ability of uric acid; (2) The body's PH value decreased caused by the increased of acidic metabolites such as lactic acid, affecting the body's uric acid excretion. Although uric acid is considered as an antioxidant, if excessive accumulation (normal $\leq 7 \mathrm{mg} / \mathrm{dl}$ ), it may lead to the impaired of body normal function, and result in high uric acid inflammatory disease.

In this study, the increased uric acid level in experimental group was significantly lower than the control group $(\mathrm{P}<0.05)$. The serum uric acid in the experimental group was $410.45 \pm 71.87 \mathrm{uM}$, lower than maximum value. From the rising dates in table 1 , the experimental group increased slowly than control group after exercise immediately to the period of 1 hour recovery. This result suggests that astaxanthin plays a control role in the uric acid's increased after acute high-intensity exercise, and that because the astaxanthin complement can effectively improve the body's antioxidant capacity.

\section{Conclusion}

How to effectively inhibit the oxidation and prevent the oxidative damage caused by free radicals is is always interesting to study of sports medicine. In this study, presupplement of astaxanthin can accelerate the recovery of antioxidant capacity, accelerate the clearance of blood lactate and delay the increase of blood uric acid in the body within 1 hour after acute high-intensity exercise. Our study only motor and detected the serum uric acid in the period of $1 \mathrm{~h}$ recovery after acute high-intensity. We can not sure when the blood uric acid level can reach peak point, and how long it will return to the quiet state, and this is the issue need to 
continue study.

\section{Acknowledgements}

The authors would like to thank all the athletes who have contributed with time and openness related to use of dietary supplements. The authors thank those who contributed to the development of this research.

\section{Funding}

The project described was supported by the Key Research and Development (R \& D) Projects of Shanxi Province (No.: 201803D31030), The content is solely the responsibility of the authors. We thank the Key Research and Development (R $\&$ D) Projects of Shanxi Province for funding this research.

\section{Scientific Contributions of Each Author}

Lijun WU Prof:: First author, substantial contributions to conception and design, drafting and revising the manuscript and confirmation of final version to be published.

Zhuo SUN (Master): Revising the manuscript and confirmation of final version to be published and Submitted manuscript.

Jing ZHAO (Master): Performed sample collection.

Xinming GUO A/Prof.: Conceived and designed the experiments.

Jie WANG (Master): Performed sample collection.

\section{Conflicts of Interest}

The authors certify that there is no conflict of interest with any financial organization regarding the material discussed in the manuscript.

\section{References}

[1] Bigarella, C. L., Liang R., Ghaffar, S. (2014.) Stem cells and the impact of ROS signaling. Development. 141 (22): 42064218 .

[2] Andersent, L. P., Holck, S., Kupcinskas, L., Kiudelis, G., Jonaitis, L., Janciauskas, D., Permin, Wadstr, M. T. (2013). Gastric inflammatory markers and interleukins in patients with functional dyspepsia treated with astaxanthin. Pathogens \& Disease. 50 (2): 244-248.

[3] HÖhn, A., KÖnig, J., Grune, T. (2013). Protein oxidation in aging and the removal of oxidized proteins. Journal of Proteomics. 92, 132-159.
[4] Ambati, R. R., Siew, M. P., Ravi, S. (2014) Astaxanthin: Sources, Extraction, Stability, Biological Activities and Its Commercial Applications-A Review. Marine Drugs 12 (1): 128-152.

[5] Polotow, T. G., Vardaris, C. V., Mihaliuc, A. R., Gonçalves, M. S., Pereira, Ganini., Barros, M. P. (2014) Astaxanthin Supplementation Delays Physical Exhaustion and Prevents Redox Imbalances in Plasma and Soleus Muscles of Wistar Rats. Nutrients. 6 (12): 5819-5838.

[6] Fumio, S., TakayaA, O., Mutsuki, I. (2015). Effects of Daily Astaxanthin and L-carnitine Supplementation for Exercise Induced Muscle Damage in Training Thoroughbred Horses. Journal of Equine Veterinary Science. 35 (10): 836-842.

[7] Sophia, Z. L., Amir, S. A., Matthew, D. Campbell., Kevin, K., Shankland, E. G., Baback, R., David, J. M., Kevin, E. C. (2018). Building strength, endurance, and mobility using an astaxanthin formulation with functional training in elderly. J Cachexia Sarcopenia Muscle. 9 (5): 826-833.

[8] Ayano, I., Yuriko, O., Naoki, T., Shinobu, S., Kiyotaka, N., Teruo, M., Fumitaka, U. (2018). Effects of Dietary Supplementation of Astaxanthin and Sesamin on Daily Fatigue: A Randomized, Double-Blind, Placebo -Controlled, Two-Way Crossover Study. Nutrients. 10 (3): 281.

[9] Maki, T., Wataru, A., Hitomi, T., Yuko, T., Sayori, W., Akane, H. (2019). Effect of dietary antioxidant-rich foods combined with aerobic training on energy metabolism in healthy young men. J Clin Biochem Nutr. 64 (1): 79-85.

[10] Wu Lijun, Guo Xinming, Zhang Junfeng. (2008). Effect of lycopene and exercise on the metabolism of free radicals in human serum. sports science. 28 (2): 47-53.

[11] Lijun Wu, Zhuo Sun, An'ping Chen, Xinming Guo, Jie Wang. (2018). Effect of astaxanthin and exercise on antioxidant capacity of human body, blood lactic acid and blood uric acid metabolism. Science \& Sports. doi.org/10.1016/j.scispo.2018.12.008.

[12] Katrin, P., Michael, R., Katrin, S., Kathrin, R. S., Marina, K. (2015). Lactic acid delays the inflammatory response of human monocytes. Biochemical and Biophysical Research Communications. 457: 412-418.

[13] Yuichiro Nishida, Minko Iyadomi, Yasuki Higai, Hiroaki Tanaka, Megumi Hara, Keitaro Tanka. (2011). Influence of Physical Activity Intensity and Aerobic Fitness on the Anthropometric Index and Serum Uric Acid Concentration in People with Obesity. Internal Medicine. 50: 2121-2128.

[14] Lamina, S., Okoye, G. C. (2012). Effects of Aerobic Exercise Training on Psychosocial Status and Serum Uric Acid in Men with Essential Hypertension: A Randomized Controlled Trial. Annals of Medical and Health Sciences Research. 2 (2): 161-168.

[15] Li-ling HUANG, Chien-Tsai HUANG, Mei-lien CHEN, IFang Mao. (2010). Effects of Profuse Sweating Induced by Exercise on Urinary Uric Acid Excretion in a Hot Environment. Chinese Journal of Physiology. 53 (4): 254-261. 